\title{
A scoping review of cost-effectiveness of screening and treatment for latent tuberculosis infection in migrants from high-incidence countries
}

\author{
Lorenzo Zammarchi ${ }^{1}$, Gianluigi Casadei ${ }^{2}$, Marianne Strohmeyer ${ }^{1}$, Filippo Bartalesi ${ }^{3}$, Carola Liendo ${ }^{4}$, \\ Alberto Matteelli ${ }^{5}$, Maurizio Bonati ${ }^{2}$, Eduardo Gotuzzo ${ }^{4}$, Alessandro Bartoloni ${ }^{1,3^{*}}$ and the COHEMI project
}

study group

\begin{abstract}
Background: In low-incidence countries, most tuberculosis (TB) cases occur among migrants and are caused by reactivation of latent tuberculosis infection $(L T B I)$ acquired in the country of origin. Diagnosis and treatment of LTBI are rarely implemented to reduce the burden of TB in immigrants, partly because the cost-effectiveness profile of this intervention is uncertain.

The objective of this research is to perform a review of the literature to assess the cost-effectiveness of LTBI diagnosis and treatment strategies in migrants.
\end{abstract}

Methods: Scoping review of economic evaluations on LTBI screening strategies for migrants was carried out in Medline.

Results: Nine studies met the inclusion criteria. LTBI screening was cost-effective according to seven studies. Findings of four studies support interferon gamma release assay as the most cost-effective test for LTBI screening in migrants. Two studies found that LTBI screening is cost-effective only if carried out in immigrants who are contacts of active TB cases.

Discussion and Conclusions: Our findings support the cost-effectiveness of LTBI diagnostic and treatment strategies in migrants especially if they are focused on young subjects from high incidence countries. These strategies could represent and adjunctive and synergistic tool to achieve the ambitious aim of TB elimination.

\section{Background}

In low incidence countries, the majority of the cases of active tuberculosis (TB) occur among migrants from high incidence countries [1]. In this setting migrants can develop TB following three main mechanisms [2]:

1. TB can already be present at the time they enter in the host country;

\footnotetext{
* Correspondence: alessandro.bartoloni@unifi.it

${ }^{1}$ Infectious Diseases Unit, Department of Experimental \& Clinical Medicine, University of Florence School of Medicine, Largo Brambilla 3, 50134 Florence, Italy

${ }^{3}$ SOD Malattie Infettive e Tropicali, Azienda Ospedaliero-Universitaria Careggi, Largo Brambilla 3, 50134 Florence, Italy

Full list of author information is available at the end of the article
}

2. TB can be the consequence of the reactivation of latent tuberculosis infection (LTBI) acquired in the country of origin, occurring months to years after the settlement in the host country $[3,4]$;

3. Primary progressive TB can follow a new infection acquired in the host country $[4,5]$ or during a return travel to the country of origin [6].

Most countries with low TB incidence adopt TB screening policies for migrants from high $\mathrm{TB}$ incidence countries. The majority of countries screen migrants for active TB through chest $\mathrm{x}$-ray (CXR) before or soon after arrival, while screening for LTBI is not consistently imple mented [7]. Screening protocols that include CXR as first 
step are able to identify the majority of migrants with active TB at entry and, occasionally, migrants with radiological alterations suggestive of LTBI. However, most persons with LTBI go undetected as a diagnostic test for this condition is not usually applied. Epidemiological studies based on molecular techniques to genotype the M. tuberculosis isolates showed that 55-90\% of TB cases diagnosed in foreign born patients are due to LTBI reactivation $[8,9]$.

Screening migrants for LTBI and providing treatment to those with this condition is a plausible strategy to prevent the disease and reduce the risk of spread infection in the native population $[10,11]$. Diagnosis and treatment of LTBI was recommended in Europe already more than ten years ago $[12,13]$, and is now included among the main interventions of the new global post-2015 strategy for TB control [13].

Only 16 of 29 industrialized countries belonging to the Organization for Economic Co-operation and Development, screen immigrants for LTBI, most frequently postarrival in the host country [7].

In 11 of these 16 countries, the screening is compulsory for legal migrants. Children and young adults ( $<40$ years) are most commonly targeted for LTBI screening. The most common test used for screening is Tuberculin Skin Test (TST), used in 11 out of 16 countries. Patients and physicians compliance to the LTBI screening protocol is essential for the effectiveness [10], but it is reported to be low [11].

Appropriate information on cost-effectiveness of LTBI screening strategies may help the policy makers to decide appropriate interventions. Thus, we performed a review of published economic evaluations (EE) of different LTBI screening strategies.

\section{Methods}

Ethical approval was not required for this review study.

\section{Inclusion criteria}

In this review we included studies with all the three following criteria: 1) had migrants as study target population; 2) included diagnosis and treatment for LTBI; 3) reported findings of EE analyses. Both model-based EEs and those alongside clinical trials (or in combinations as well) were included.

\section{Search strategy}

MEDLINE and the Cochrane Library electronic databases were searched for studies published up to July 2014. The terms used for the search strategy were: (latent tuberculosis OR LTBI OR "latent tuberculosis"[Mesh]) AND (screening OR Mantoux OR IGRA OR "mass screening"[Mesh] OR "tuberculin test"[Mesh] OR "Interferongamma Release Tests”[Mesh]) AND (cost-effect* OR cost-bene* OR "Cost- Benefit Analysis"[Mesh]). No language restriction was done. The search was performed also using the term "latent tuberculosis screening in migrants" as free text.

\section{Data extraction and assessment}

All references retrieved were collected using the EndNote, version X5 (Thomson Reuter) program. Identified titles and abstracts were screened for their eligibility for inclusion and the full text of potentially relevant studies was obtained and examined. Two review authors (LZ, GC) independently screened titles and abstracts of each study. Based on the full text revision, the two reviewers independently selected the studies, and inter-reviewer disagreement was solved by discussion. The following information was extracted: setting, study design, participants, EEs data, including type of EE, screening alternatives, cost description, analysis perspective, source of data (literature, clinical studies), modeling (if any, including time horizon and discount rate), key results and authors' conclusions. The following data were collected for clinical trials used for EE analyses: inclusion criteria, study participants, setting, design and methods, results and authors' conclusions. Agreement on inclusion was calculated using the Kappa statistics.

Study quality was assessed using an established checklist of criteria for assessing the quality of economic evaluations in health care [14]. This tool includes ten items regarding cost effectiveness analysis: the presence of a clearly stated hypothesis and comparator; the used methods; the medical evidence; appropriate costs and benefits considered; a marginal analysis and a sensitivity analysis have been undertaken; the analysis was appropriate to the local environment.

\section{Results}

Search results

The literature search resulted in 109 titles. A total of 86 duplicates or non-pertinent or non-appropriate references were deleted, resulting in 23 potentially relevant studies. Reviewers agreed on 18 of 23 papers $(78.3 \%)$ selected for reliability check $(K=0.697)$, and disagreements were resolved by consensus. Ten of 23 papers $(43.5 \%)$ met inclusion criteria and were therefore included in the final step of review (Fig. 1).

\section{General characteristics of included studies}

The 10 research studies included in the review (Table 1) were published in 8 journals, 2 in the Am J Respir Crit Care Med and Thorax, and the others in 6 different journals. The distribution of papers per year of publication ranged from 2000 to 2014.

Four studies were performed in the US, 3 in the United Kingdom, and 3 in Canada. 


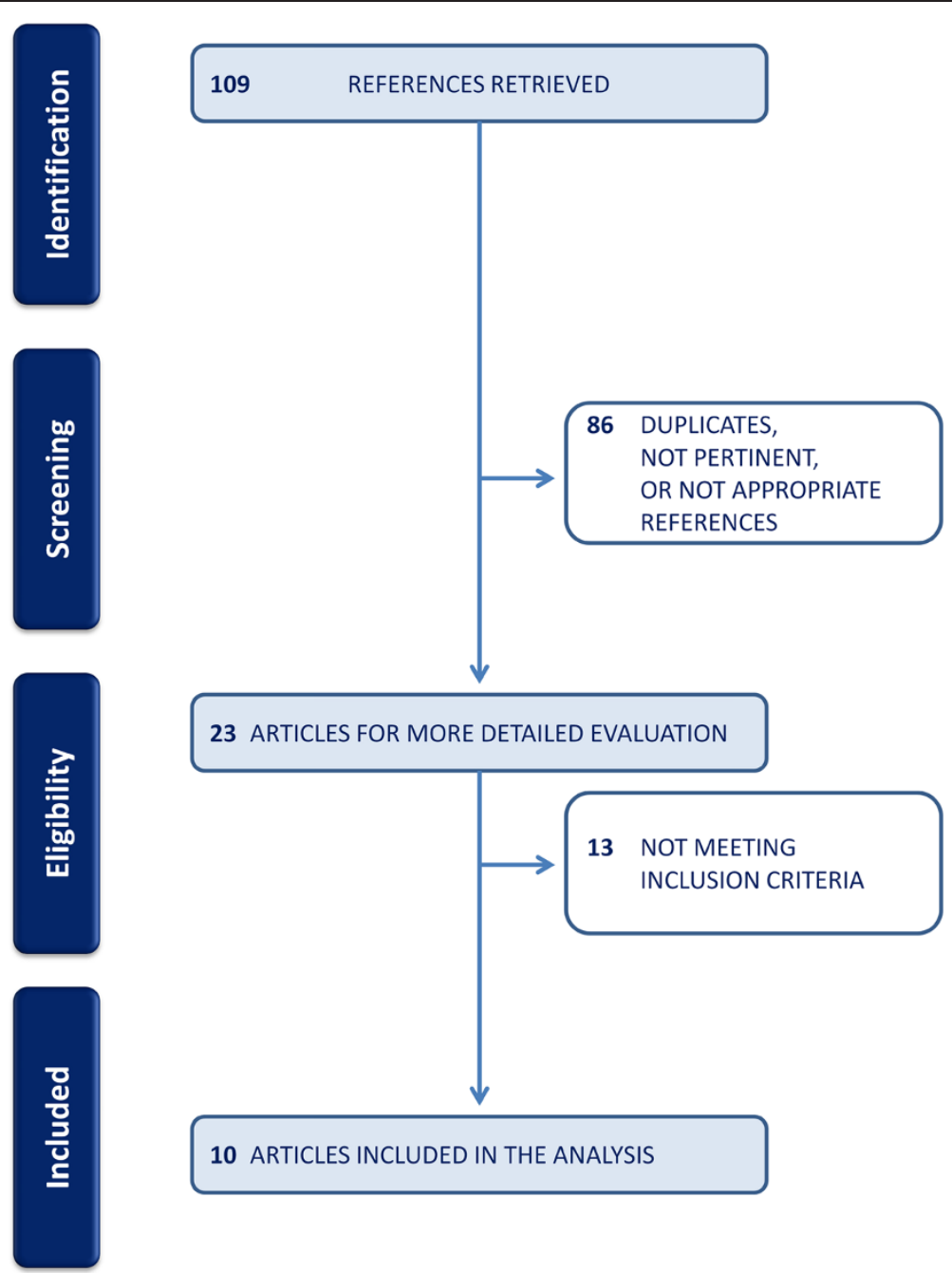

Fig. 1 Flow diagram showing the number of papers identified by the search and the selection process

In four studies age limitations in the study population were used: children 4 to 18 years old [17], migrants $>16$ year old (median 29 years) [16], migrants or foreign born subjects $>18$ year old [18], and migrants aging $<35$ years [15].

Four studies considered immigrants at the moment of their entrance or application for residence permission $[11,18-20]$. Two studies referred to "new immigrants" or foreign born subjects without further specification $[17,21,22]$. Two studies referred to immigrants arrived within 5 years $[7,15]$. One study considered both recent migrants and foreign born residents in US for more than 5 years [23].

In 5 studies the screening was carried out according to TB incidence in the country of origin regardless of age $[11,17,19,20,23]$, whereas 3 studies compared the cost-effectiveness of screening migrants with different thresholds of TB incidence in the country of origin
$[14,16,21]$. One study considered migrants from all developing countries [18].

Several LTBI diagnostic strategies (screening with CXR and follow-up of inactive TB, screening with TST, screening with TST followed by confirmation with Interferon gamma release assay - IGRA, screening with single step IGRA) were compared with each other and/or with different nonLTBI screening strategies including no screening, screening for active TB with CXR, and close-contact investigation (Table 1). Isoniazid for 6-9 months was the LTBI treatment regimen used in the majority of studies $[11,17,19,20,22$, 23 ], in one study isoniazid plus rifampicin for 3 months was used [16], in one study either isoniazid for 6 months or isoniazid plus rifampicin for 3 months were used [15], and in one study different LTBI treatment regimens (isoniazid for 9 months, rifampicin for 4 months, rifampicin plus pyrazinamide for 2 months) were compared [18]. In one study the LTBI treatment regimen was not specified [21]. 
Table 1 Economic evaluations of latent tuberculosis infection screenings published up to July 2014

\begin{tabular}{|c|c|c|c|c|c|c|c|c|c|c|}
\hline $\begin{array}{l}\text { Author } \\
\text { (Country; Year) }\end{array}$ & Alternatives & Data source & $\begin{array}{l}\text { EE } \\
\text { Type }\end{array}$ & Perspective & $\begin{array}{l}\text { Model } \\
\text { Horizon } \\
\text { Discount } \\
\text { rate }\end{array}$ & Cost & Cost description & Differential Costs & ICER & Conclusion \\
\hline \multirow{4}{*}{$\begin{array}{l}\text { Dasgupta et al. } \\
\text { (Canada; 2000) } \\
\text { [19] }\end{array}$} & $\begin{array}{l}\text { 1. Screening (medical } \\
\text { evaluation, CXR); }\end{array}$ & \multirow[t]{4}{*}{$\begin{array}{l}\text { Administrative } \\
\text { data bases }\end{array}$} & \multirow[t]{4}{*}{ CEA } & \multirow[t]{4}{*}{$\begin{array}{l}\text { Health care } \\
\text { payer }\end{array}$} & \multirow[t]{4}{*}{$\begin{array}{l}\text { Markov } \\
20 \text { years }\end{array}$} & \multirow[t]{4}{*}{ Direct } & \multirow{4}{*}{$\begin{array}{l}\text { CXR, clinic visits, } \\
\text { investigations, } \\
\text { hospitalization, } \\
\text { drugs, physicians' and } \\
\text { pharmacists' fees, } \\
\text { administrative } \\
\text { activities. }\end{array}$} & \multirow{4}{*}{$\begin{array}{l}\text { - TB case detected: } \\
\text { CAN\$ } 31,418 ; \\
\text { prevented: } \\
\text { CAN\$ } 73,125 ; \\
\text { - CAN\$ 55,728; } \\
\text { CAN\$ 155,729; } \\
\text { - CAN\$ 10,275; } \\
\text { CAN\$ } 29,668\end{array}$} & & \multirow{4}{*}{$\begin{array}{l}\text { Close-contact } \\
\text { investigation was highly } \\
\text { cost-effective and } \\
\text { resulted in net savings. } \\
\text { Immigrant applicant } \\
\text { screening and } \\
\text { surveillance programs } \\
\text { had a significant impact } \\
\text { but were much less } \\
\text { cost-effective, in large } \\
\text { part because of } \\
\text { substantial operational } \\
\text { problems. Radiographic } \\
\text { screening of newly } \\
\text { arriving foreign-born } \\
\text { populations for TB } \\
\text { could be cost-effective } \\
\text { and have considerable } \\
\text { individual and public } \\
\text { health benefits. }\end{array}$} \\
\hline & 2. Surveillance of inactive & & & & & & & & & \\
\hline & $\begin{array}{l}\mid \mathrm{B} \text {, including } \mathrm{LIB|} \\
\text { treatment; }\end{array}$ & & & & & & & & & \\
\hline & $\begin{array}{l}\text { 3. Close-contact } \\
\text { investigation }\end{array}$ & & & & & & & & & \\
\hline \multirow{9}{*}{$\begin{array}{l}\text { Khan et al. } \\
\text { (USA; 2002) } \\
{[18]}\end{array}$} & 1. No LTBI screening; & \multirow[t]{9}{*}{ Data bases } & \multirow{9}{*}{$\begin{array}{l}\text { CEA, } \\
\text { CUA }\end{array}$} & \multirow[t]{9}{*}{ Societal } & \multirow{9}{*}{$\begin{array}{l}\text { Markov } \\
\text { Lifetime } \\
3.00 \%\end{array}$} & \multirow{9}{*}{$\begin{array}{l}\text { Health } \\
\text { Direct }\end{array}$} & \multirow{9}{*}{$\begin{array}{l}\text { Transportation, } \\
\text { ambulatory care, } \\
\text { services of } \\
\text { interpreters, } \\
\text { laboratory tests, } \\
\text { medications, adverse } \\
\text { drug reactions, } \\
\text { hospitalization, and } \\
\text { patients' time. }\end{array}$} & \multirow{9}{*}{$\begin{array}{l}\text { Total savings: US\$60 } \\
\text { to US\$90 million, } \\
\text { assuming to avert } \\
9-10 \text { thousand TB } \\
\text { infections per year. }\end{array}$} & CEA & \multirow{2}{*}{$\begin{array}{l}\text { Results variable } \\
\text { according to country } \\
\text { of origin. }\end{array}$} \\
\hline & \multirow{8}{*}{$\begin{array}{l}\text { 2. TST followed by INH; } \\
\text { 3. TST followed by RIF; } \\
\text { 4. TST followed by RIF } \\
\text { plus PZM. }\end{array}$} & & & & & & & & - TST followed by INH: & \\
\hline & & & & & & & & & depending on regions. & \multirow{3}{*}{$\begin{array}{l}\text { A strategy of } \\
\text { detecting and } \\
\text { treating LTBI was } \\
\text { cost-saving among } \\
\text { immigrants from } \\
\text { Mexico, Haiti, sub- } \\
\text { Saharan Africa, South } \\
\text { Asia, and developing } \\
\text { nations in East Asia } \\
\text { and the Pacific. }\end{array}$} \\
\hline & & & & & & & & & $\begin{array}{l}\text { - TST followed RIF: } \\
\text { dominant. }\end{array}$ & \\
\hline & & & & & & & & & $\begin{array}{l}\text { - TST followed RIF plus } \\
\text { PZM: saving or cost/ } \\
\text { effective (US } \$ 2,551 \text { - } \\
\text { US\$149,978 per future } \\
\text { case averted), } \\
\text { depending on regions. }\end{array}$ & \\
\hline & & & & & & & & & per QALY & \multirow{4}{*}{$\begin{array}{l}\text { Screening was highly } \\
\text { cost-effective among } \\
\text { immigrants from } \\
\text { other developing } \\
\text { nations. RIF-PZM was } \\
\text { the preferred } \\
\text { treatment for treating } \\
\text { LTBI in immigrants } \\
\text { from Vietnam, Haiti, } \\
\text { and the Philippines. }\end{array}$} \\
\hline & & & & & & & & & $\begin{array}{l}\text { - TST followed by INH: } \\
\text { savings, US\$914 - } \\
\text { US\$5,952, or dominated } \\
\text { depending on regions. }\end{array}$ & \\
\hline & & & & & & & & & $\begin{array}{l}\text { - TST followed by RIF: } \\
\text { dominant. }\end{array}$ & \\
\hline & & & & & & & & & $\begin{array}{l}\text { - TST followed by RIF } \\
\text { plus PZM: savings, or } \\
\text { US\$1,276 - US\$53,388, } \\
\text { depending on regions. }\end{array}$ & \\
\hline
\end{tabular}


Table 1 Economic evaluations of latent tuberculosis infection screenings published up to July 2014 (Continued)

\begin{tabular}{|c|c|c|c|c|c|c|c|c|c|c|}
\hline \multirow{3}{*}{$\begin{array}{l}\text { Brassard et al. } \\
\text { (Canada; 2006) } \\
\text { [17] }\end{array}$} & \multirow{2}{*}{$\begin{array}{l}\text { 1. LTBI school-screening } \\
\text { program in newly arrived } \\
\text { immigrant children (TST } \\
\text { followed by INH); }\end{array}$} & \multirow[t]{3}{*}{ Clinical trial $^{c}$} & \multirow[t]{3}{*}{ CBA } & \multirow[t]{3}{*}{$\begin{array}{l}\text { Health care } \\
\text { payer }\end{array}$} & \multirow[t]{3}{*}{ No } & \multirow[t]{3}{*}{$\begin{array}{l}\text { Health } \\
\text { Direct }\end{array}$} & \multirow{3}{*}{$\begin{array}{l}\text { Total material and } \\
\text { labor costs associated } \\
\text { with the school- } \\
\text { screening program } \\
\text { and the associate } \\
\text { investigations for } \\
\text { children and } \\
\text { associates. }\end{array}$} & \multicolumn{2}{|l|}{$\begin{array}{l}\text { Total savings: } \\
\text { CAN\$ } 363,923 \text {. }\end{array}$} & \multirow{2}{*}{$\begin{array}{l}\text { The school-based } \\
\text { LTBI-screening } \\
\text { program was found } \\
\text { to be cost-effective. }\end{array}$} \\
\hline & & & & & & & & \multirow{2}{*}{\multicolumn{2}{|c|}{$\begin{array}{l}\text { Without associate } \\
\text { investigation: } \\
\text { CAN\$ 268,393. }\end{array}$}} & \\
\hline & $\begin{array}{l}\text { 2. Active TB } \\
\text { management through } \\
\text { passive case finding. }\end{array}$ & & & & & & & & & $\begin{array}{l}\text { Savings were mainly } \\
\text { due to hospitalization } \\
\text { costs. }\end{array}$ \\
\hline \multirow{3}{*}{$\begin{array}{l}\text { Porco et al. } \\
\text { (USA; 2006) } \\
{[20]}\end{array}$} & $\begin{array}{l}\text { 1. Follow-up of TB- } \\
\text { notification patients, }\end{array}$ & \multirow{3}{*}{$\begin{array}{l}\text { Published } \\
\text { literature, } \\
\text { administrative } \\
\text { data bases }\end{array}$} & \multirow[t]{3}{*}{$\begin{array}{l}\text { CEA, } \\
\text { CUA }\end{array}$} & \multirow[t]{3}{*}{$\begin{array}{l}\text { Health care } \\
\text { payer }\end{array}$} & \multirow{3}{*}{$\begin{array}{l}\text { Markov } \\
20 \text { years } \\
3.00 \%\end{array}$} & \multirow[t]{3}{*}{ Direct } & \multirow{3}{*}{$\begin{array}{l}\text { Diagnostic tests, } \\
\text { nursing assessments } \\
\text { and doctor visits, } \\
\text { drugs, side effects, } \\
\text { hospitalizations. }\end{array}$} & \multirow{3}{*}{$\begin{array}{l}\text { The program yielded } \\
7.7 \text { net QALYs, US\$ } \\
25,000 \text { in net savings, } \\
\text { and prevented } 4 \\
\text { cases of TB. }\end{array}$} & $\begin{array}{l}\text { Treatment of TB4s } \\
\text { was cost-saving. }\end{array}$ & \multirow{3}{*}{$\begin{array}{l}\text { Domestic follow up is } \\
\text { highly cost-effective } \\
\text { as early detection and } \\
\text { treatment reduces the } \\
\text { rate of hospitalization. }\end{array}$} \\
\hline & $\begin{array}{l}\text { including } \mathrm{LIBI} \text { treatment } \\
\text { for latently infected } \\
\text { individuals; }\end{array}$ & & & & & & & & \multirow{2}{*}{$\begin{array}{l}\text { Treatment of TB2s } \\
\text { was highly cost- } \\
\text { effective: US\$4,400 } \\
\text { per QALY and } \\
\text { US\$4,700 per case } \\
\text { prevented. }\end{array}$} & \\
\hline & 2. No follow-up. & & & & & & & & & \\
\hline \multirow{11}{*}{$\begin{array}{l}\text { Oxlade O et al. } \\
\text { (Canada;2007) } \\
\text { [11] }\end{array}$} & 1. $C X R$ & \multirow{11}{*}{$\begin{array}{l}\text { Published } \\
\text { literature, } \\
\text { administrative } \\
\text { data bases }\end{array}$} & \multirow[t]{11}{*}{ CEA } & \multirow[t]{11}{*}{ Societal } & Markov & \multirow{11}{*}{$\begin{array}{l}\text { Health } \\
\text { Direct }\end{array}$} & \multirow{11}{*}{$\begin{array}{l}\text { All government and } \\
\text { health system costs, } \\
\text { patients' out-of- } \\
\text { pocket expenditures, } \\
\text { but not TB-related } \\
\text { death or disability. }\end{array}$} & \multirow{5}{*}{$\begin{array}{l}\text { Savings only in } \\
\text { high-very high risk } \\
\text { populations: }\end{array}$} & \multirow{5}{*}{$\begin{array}{l}\text { CXR - the least costly } \\
\text { ICER per case } \\
\text { prevented: CAN\$ } 875 \\
\text { for immigrants from } \\
\text { high-incidence TB up } \\
\text { to CAN\$ } 2.2 \text { million } \\
\text { from low incidence. }\end{array}$} & \multirow{3}{*}{$\begin{array}{l}\text { Screening with CXR } \\
\text { would be the most } \\
\text { and QFT the least } \\
\text { cost-effective. }\end{array}$} \\
\hline & 2. TST; & & & & 20 years & & & & & \\
\hline & 3. $\mathrm{QFT}$ & & & & $3.00 \%$ & & & & & \\
\hline & 4. TST+QTF; & & & & & & & & & Screening for $L T B I$, with \\
\hline & 5. No screening. & & & & & & & & & effective only if the risk \\
\hline & & & & & & & & $\begin{array}{l}\cdot \text { TST - CAN\$ 136260; } \\
\text { CAN\$ 476320; }\end{array}$ & $\begin{array}{l}\text { QFT - the most } \\
\text { expensive: }\end{array}$ & \\
\hline & & & & & & & & $\begin{array}{l}\text { - QFT - CAN\$ 100,490; } \\
\text { CAN\$ 440,550. }\end{array}$ & $\begin{array}{l}\text { CAN\$62,643 up to } \\
\text { CAN\$1,122,200. }\end{array}$ & $\begin{array}{l}\text { of QFT is to test TST- } \\
\text { positive persons. }\end{array}$ \\
\hline & Three scenarios: & & & & & & & & TST - better than QFT & Screening with TST or \\
\hline & $\begin{array}{l}\text { a) immigration entry } \\
\text { screening; }\end{array}$ & & & & & & & & CAN $\$ 35,000$ & cost-effective in \\
\hline & b) close or & & & & & & & & in populations BCG- & entering immigrants. \\
\hline & c) casual contacts. & & & & & & & & $\begin{array}{l}\text { vaccinated after } \\
\text { infancy, where TST } \\
\text { more expensive } \\
\text { because of low } \\
\text { specificity. }\end{array}$ & $\begin{array}{l}\text { However, the } \\
\text { selection of screening } \\
\text { strategy is less } \\
\text { important than } \\
\text { program } \\
\text { performance. } \\
\text { Programs considering } \\
\text { these new ex vivo tests } \\
\text { for LTBI should thus } \\
\text { first ensure that a high } \\
\text { proportion of those } \\
\text { with positive tests will } \\
\text { be medically } \\
\text { evaluated, prescribed } \\
\text { and complete therapy. }\end{array}$ \\
\hline
\end{tabular}


Table 1 Economic evaluations of latent tuberculosis infection screenings published up to July 2014 (Continued)

\begin{tabular}{|c|c|c|c|c|c|c|c|c|c|c|}
\hline \multirow{3}{*}{$\begin{array}{l}\text { Hardy et al. } \\
\text { (UK; 2010) } \\
\text { [21] }\end{array}$} & \multirow{3}{*}{$\begin{array}{l}\text { 1. NICE guidance 2006; } \\
\text { 2. Leeds protocol: QTF } \\
\text { first in immigrants from } \\
\text { countries with TB } \\
\text { incidence }>200 / 10^{5} \\
\text { followed by CXR (all ages, } \\
\text { but mean age was } 30.8 \\
\text { year). }\end{array}$} & \multirow[t]{3}{*}{ Clinical trial $^{c}$} & \multirow[t]{3}{*}{ CEA } & \multirow[t]{3}{*}{$\begin{array}{l}\text { Health care } \\
\text { payer }\end{array}$} & \multirow[t]{3}{*}{ No } & \multirow{3}{*}{$\begin{array}{l}\text { Health } \\
\text { Direct }\end{array}$} & \multirow{3}{*}{$\begin{array}{l}\text { Cost per case LTBI } \\
\text { identified }\end{array}$} & \multirow{3}{*}{\multicolumn{2}{|c|}{$\begin{array}{l}\text { Cost per case } \\
\text { identified: } \\
\text { • NICE protocol: £160.81; } \\
\text { • Leed protocol: £93.16. }\end{array}$}} & \multirow{3}{*}{$\begin{array}{l}\text { QFT-first protocol can } \\
\text { be carried out more } \\
\text { cheaply than a CXR- } \\
\text { first protocol, with a } \\
\text { cost-saving of about } \\
35 \% \text { ( } £ 67.65) \\
\text { compared to NICE } \\
\text { protocol. This saving } \\
\text { is due to the reduced } \\
\text { number of CXRs } \\
\text { required. }\end{array}$} \\
\hline & & & & & & & & & & \\
\hline & & & & & & & & & & \\
\hline \multirow{7}{*}{$\begin{array}{l}\text { Linas et al. } \\
\text { (USA; 2011) } \\
\text { [23] }\end{array}$} & 1. TST; & \multirow{7}{*}{$\begin{array}{l}\text { Published } \\
\text { literature }\end{array}$} & \multirow{7}{*}{$\begin{array}{l}\text { CEA, } \\
\text { CUA }\end{array}$} & \multirow{7}{*}{$\begin{array}{l}\text { Health care } \\
\text { payer }\end{array}$} & \multirow{7}{*}{$\begin{array}{l}\text { Markov } \\
\text { Lifetime } \\
3.00 \%\end{array}$} & \multirow{7}{*}{$\begin{array}{l}\text { Health } \\
\text { Direct }\end{array}$} & \multirow{7}{*}{$\begin{array}{l}\text { Nursing and physician } \\
\text { visits, diagnostic tests, } \\
\text { medications, } \\
\text { hospitalizations, } \\
\text { contact tracing, and } \\
\text { directly observed } \\
\text { therapy. }\end{array}$} & & \multirow{4}{*}{$\begin{array}{l}\text { - Individuals at } \\
\text { highest risk of TB } \\
\text { reactivation (close } \\
\text { contacts and HIV- } \\
\text { infected) - ICER of } \\
\text { IGRA compared to } \\
\text { TST was }<\$ 100,000 \text { / } \\
\text { QALY gained. }\end{array}$} & \multirow{7}{*}{$\begin{array}{l}\text { In foreign-born } \\
\text { subjects IGRA was } \\
\text { cost-saving compared } \\
\text { to TST and cost- } \\
\text { effective compared to } \\
\text { no screening. }\end{array}$} \\
\hline & 2. IGRA; & & & & & & & & & \\
\hline & 3. No LTBI screening. & & & & & & & & & \\
\hline & Risk-groups $^{a}$ & & & & & & & & & \\
\hline & & & & & & & & & $\begin{array}{l}\text { - The foreign-born - } \\
\text { IGRA was cost-saving } \\
\text { compared to TST and } \\
\text { cost-effective compared } \\
\text { to no screening (ICER } \\
<\$ 100,000 / \text { QALY } \\
\text { gained). }\end{array}$ & \\
\hline & & & & & & & & & $\begin{array}{l}\text {-Vulnerable populations } \\
\text { (homeless, drug user, } \\
\text { former prisoner) - ICER } \\
\text { of TST screening was } \\
\text { approximately } \\
\$ 100,000-\$ 150,000 / \\
\text { QALY gained, but IGRA } \\
\text { was not cost-effective. }\end{array}$ & \\
\hline & & & & & & & & & $\begin{array}{l}\text { - Medical co-morbidities } \\
\text { (diabetes and others) - } \\
\text { ICER of screening with } \\
\text { TST or IGRA was } \\
>\$ 100,000 / \text { QLLY. }\end{array}$ & \\
\hline
\end{tabular}


Table 1 Economic evaluations of latent tuberculosis infection screenings published up to July 2014 (Continued)

\begin{tabular}{|c|c|c|c|c|c|c|c|c|c|c|}
\hline \multirow{4}{*}{$\begin{array}{l}\text { Pareek et al. } \\
\text { (UK; 2011) } \\
{[15]}\end{array}$} & 1. QFT; & \multirow[t]{4}{*}{ Clinical trial $^{c}$} & \multirow[t]{4}{*}{ CEA } & \multirow{4}{*}{$\begin{array}{l}\text { Government } \\
\text { health care } \\
\text { payer }\end{array}$} & \multirow{4}{*}{$\begin{array}{l}\text { Markov } \\
20 \text { years } \\
3.50 \%\end{array}$} & \multirow[t]{4}{*}{ Direct } & \multirow[t]{4}{*}{ UK NICE TB guidelines } & Screening of & - Screen immigrants & \multirow{4}{*}{$\begin{array}{l}\text { Screening for latent } \\
\text { infection can be } \\
\text { implemented cost- } \\
\text { effectively at a level of } \\
\text { incidence that } \\
\text { identifies most } \\
\text { immigrants with } \\
\text { latent tuberculosis, } \\
\text { thereby preventing } \\
\text { substantial numbers } \\
\text { of future cases of } \\
\text { active tuberculosis. }\end{array}$} \\
\hline & \multirow[t]{3}{*}{ 2. NICE guidance $2006^{b}$. } & & & & & & & $\begin{array}{l}\text { countries irrespective } \\
\text { of tuberculosis } \\
\text { incidence would cost: }\end{array}$ & $\begin{array}{l}\text { countries with } \\
\text { incidences } / 10^{5}\end{array}$ & \\
\hline & & & & & & & & $\begin{array}{l}\text { - QFT more than } £ 1.5 \\
\text { million and prevent } \\
44.5 \text { cases of } \\
\text { tuberculosis; }\end{array}$ & $\begin{array}{l}\geq 250 / 10^{5}: £ 17,956 \\
\text { per case averted; } \\
\geq 150 / 10^{5}: £ 20,819 ; \\
\geq 40 / 10^{5}: £ 29,403 \text {. }\end{array}$ & \\
\hline & & & & & & & & $\begin{array}{l}\text { - NICE guidance } \\
\approx £ 850,000 \text { and } \\
\text { prevent } 13.2 \text { cases. }\end{array}$ & $\begin{array}{l}\text { Screen immigrants } \\
\text { aged } \leq 35 \text { years } \\
\text { irrespective of TB } \\
\text { incidence: } £ 101,938 \text {. }\end{array}$ & \\
\hline \multirow{5}{*}{$\begin{array}{l}\text { Pareek et al. } \\
\text { (UK; 2012) } \\
{[16]}\end{array}$} & $\begin{array}{l}\text { 1. TST+ in <35y } \\
\text { old immigrants; }\end{array}$ & \multirow[t]{5}{*}{ Clinical trial $^{\mathrm{C}}$} & \multirow[t]{5}{*}{ CEA } & \multirow{5}{*}{$\begin{array}{l}\text { Government } \\
\text { health care } \\
\text { payer }\end{array}$} & \multirow[t]{5}{*}{$\begin{array}{l}\text { Markov } \\
20 \text { years }\end{array}$} & \multirow[t]{5}{*}{ Direct } & & \multirow{5}{*}{$\begin{array}{l}\text { Current UK national } \\
\text { guidance associated } \\
\text { with additional costs } \\
\text { of between } £ 594,957 \\
\text { and } £ 1,530,303 \text { over } \\
20 \text { years. }\end{array}$} & \multirow{2}{*}{$\begin{array}{l}\text { - QFN (single): } \\
£ 21,565 \text { - } £ 34,754 \text { per } \\
\text { TB case averted. }\end{array}$} & \multirow{5}{*}{$\begin{array}{l}\text { Mandatory CXR on } \\
\text { arrival could be safely } \\
\text { eliminated in order to } \\
\text { improve screening } \\
\text { cost-effectiveness } \\
\text { with single-step QTF } \\
\text { at incidence threshold } \\
>250 / 10^{5} \text { per year. }\end{array}$} \\
\hline & $\begin{array}{l}\text { 2. QTF+ in <35y } \\
\text { old immigrants; }\end{array}$ & & & & & & & & & \\
\hline & \multirow{2}{*}{$\begin{array}{l}\text { 3. T-SPOT+ in <35y } \\
\text { old immigrants. }\end{array}$} & & & & & & & & $£ 59,489$ & \\
\hline & & & & & & & & & \multirow{2}{*}{$\begin{array}{l}\text { - CXR plus single } \\
\text { T.SPOT.TB: } £ 402,422 \text {. }\end{array}$} & \\
\hline & $\begin{array}{l}\text { All with or without CXR } \\
\text { port of entry. }\end{array}$ & & & & & & & & & \\
\hline \multirow{11}{*}{$\begin{array}{l}\text { lqbal et al. } \\
\text { (USA, 2014) [22] }\end{array}$} & 1. QFT; & \multirow{11}{*}{$\begin{array}{l}\text { Administrative } \\
\text { data base } \\
\text { (2007) }\end{array}$} & \multirow[t]{11}{*}{ CEA } & \multirow{11}{*}{$\begin{array}{l}\text { Government } \\
\text { health care } \\
\text { payer }\end{array}$} & \multirow{11}{*}{$\begin{array}{l}\text { Decision } \\
\text { model }\end{array}$} & \multirow{11}{*}{$\begin{array}{l}\text { Health } \\
\text { Direct }\end{array}$} & \multirow{11}{*}{$\begin{array}{l}\text { Screening, CXR, } \\
\text { Treatments, lab tests } \\
\text { and diagnostics, } \\
\text { physicians' and staff } \\
\text { time. }\end{array}$} & Total screening cost: & \multirow{4}{*}{$\begin{array}{l}\text { Differential costs for } \\
\text { screening and follow- } \\
\text { up for subjects who } \\
\text { were estimated to be } \\
\text { test positive on } 1,000 \\
\text { latent TB infections }\end{array}$} & \multirow{11}{*}{$\begin{array}{l}\text { QFT is cost-effective } \\
\text { especially for high-risk } \\
\text { populations such as } \\
\text { foreign-born } \\
\text { individuals. }\end{array}$} \\
\hline & 2. TST. & & & & & & & US\$74 & & \\
\hline & & & & & & & & Key assumption. & & \\
\hline & & & & & & & & False positive rates: & & \\
\hline & & & & & & & & • U.S. born & • U.S. born & \\
\hline & & & & & & & & TST 66\%; QFT 40\% & \multirow{2}{*}{$\begin{array}{l}\text { QFT: +US\$25,037 vs. } \\
\text { TST. }\end{array}$} & \\
\hline & & & & & & & & \multirow{2}{*}{$\begin{array}{l}\text { Treatment duration: } 9 \\
\text { months }\end{array}$} & & \\
\hline & & & & & & & & & \multirow{4}{*}{$\begin{array}{l}\text { •roreign Dorn } \\
\text { QFT: -US\$135,946. }\end{array}$} & \\
\hline & & & & & & & & • roreign born & & \\
\hline & & & & & & & & TST 69\%; QFT 18\% & & \\
\hline & & & & & & & & $\begin{array}{l}\text { Treatment duration: } 9 \\
\text { months }\end{array}$ & & \\
\hline
\end{tabular}

EE economic evaluation, ICER Incremental cost-effectiveness ratio, CEA cost-effectiveness analysis, CXR chest X-ray, CAN\$ Canadian dollar, INH isoniazid, RIF rifampicin, PZM pyrazinamide, CUA cost-utility analysis, US\$ United States dollar, TST tuberculin skin test, QTF Quantiferon, QALY Quality Adjusted Life Years, CBA cost-benefit analysis, LTBI latent tuberculosis infection, TB tuberculosis, NICE National Institute for Health and Care Excellence, TB2 subjects with evidence of infection but no evidence of disease, TB4 subjects with stable radiographic abnormalities suggestive of TB together with evidence of TB infection and negative bacteriologic studies

a) Risk groups including recent immigrant adults and children, foreign-born residents living in the U.S. for more than five years (stratified by age), close contact adults and children, HIV-infected individuals, the homeless, injection drug users, former prisoners, gastrectomy patients, underweight patients, and persons with silicosis, diabetes, and end-stage renal disease.

b) CXR in all immigrants from countries with TB incidence $>40 / 10^{5}$ and $>16 y$; TST if $<16 y$ o or $<35$ yo from Sub Saharan Africa or from countries $>500 / 10^{5 ;}$ QTF in TST positive to confirm LTBI

c) Clinical trial description reported as Additional file 1: Table S2 
All evaluations complied with the following quality criteria: presence of a clear hypothesis, methodology, selection of comparators, choice of appropriate benefits, and local applicability. Only four evaluations referred to clinical trials $[15-17,21]$, while medical evidence was derived from published literature in the remaining 6 studies. Appropriate direct and indirect health costs were considered in 8 assessments and sensitivity analysis was performed in seven evaluations. The lower quality study was the oldest [19]. Additional file 1: Table S1 presents different assumptions concerning progression rate and sensitivity as well as specificity of the TST and IGRAs applied in the different papers.

\section{Economic evaluations of included studies}

Cost-effectiveness analyses (CEA) were conducted in all EEs, and only one cost-benefit analysis (CBA) [16]. Costutility analysis (CUA) was performed in addition to CEA in three EEs [18, 20, 23]. EEs were conducted under the perspective of public health payer. Direct health costs were assessed in all studies while indirect costs were considered in one study [18]. As shown in Additional file 1: Table S2, four EEs were based on the results of specific studies while the remaining 6 assessments were based on available data in literature. Three studies [17, 20,23] were carried out prospectively for 12 to 31 months. Decision modeling was adopted in 8 EEs with a time horizon up to 20 years $[11,15,16,19,20]$ or lifetime $[18,23]$. Data were collected from studies carried out ad hoc in two EEs [20], while models were fed with data publicly available in the remaining 6 EEs.

Screening for LTBI in migrants was cost-effective according to 8 studies [15-18, 20-23] and cost-saving according the selected scenarios in 3 of these studies $[17,18,20]$. In 5 out of 8 studies in which LTBI screening of migrants was cost-effective, a comparison between single step IGRA and TST or TST plus confirmatory IGRA was carried out [15, 16, 22-24]. In all 5 studies one step IGRA was the most cost-effective strategy (Quantiferon ${ }^{\circ}$, QTF in 4 studies $[15,16,21,22]$ and an unspecified IGRA in the remaining study [23]). In the 2 studies in which LTBI screening strategy was not costeffective (dominated), the dominant strategies were contact tracing investigations in one case [17], and screening for active TB with CXR in the other case [11].

One article found that screening for LTBI with a single step IGRA was cost-effective regardless of time since immigration [23], while in other studies the costeffectiveness was evaluated in recent migrants only.

One study compared the cost-effectiveness of screening stratified by age and showed that screening was more cost-effective when addressing recent immigrant adults than children and that screening of US foreign-born residents aged less than 45 years was more cost-effective than screening of foreign-born residents aged 45-64 years [23]. In this study patient age affected cost-effectiveness results through its impact on the lifetime risk of reactivation. In 3 other studies, in which LTBI screening was cost-effective, the analysis was limited to children [17], or young adults $[15,16]$

Three studies compared the cost-effectiveness of screening at different thresholds of TB incidence in the country of origin. According to one study, the highest cost-effectiveness was reached by screening immigrants from countries with TB incidence above 200/100,000 while for two other studies, the threshold was 250/ $100,000[15,16,21]$.

One article provided information on which LTBI treatment regimen (among isoniazid for 9 months, rifampicin for 4 months or rifampicin plus pyrazinamide for 2 months) would be more cost-effective according to migrants country of origin showing that pyrazinamide plus rifampicin for 2 months would be the most cost-effective treatment for migrants from Vietnam, Philippines, and Haiti, while isoniazid would be the most advantageous regimen for other migrants [18].

\section{Discussion}

According to the majority of studies included in this review, at least three important elements can be underlined. First, screening programs for detecting and treating LTBI in immigrants lead to substantial health and economic benefits under a societal perspective. Second, a one-step IGRA-protocol is the most cost-effective strategy for LTBI screening in migrants. Third, targeting young migrants from countries at higher incidence of TB increases the cost-effectiveness of screening.

Limitations and possible biases should be considered. First, our review, even if performed in a systematic methodology, did not respect all the criteria for systematic reviews (for example we consulted only two electronic database) so we cannot make strong and quantitative conclusions. Secondly, we don't know whether the LTBI screening strategy that seems more cost-effective according to the majority of the selected studies (the use of IGRA) would be cost-effective in all context/jurisdiction, as this heavily depends on the country-specific treatment procedures and costs. Furthermore, we don't have the quality or quantitative weights of each study to be able to make a pooled conclusion on cost-effectiveness. All these issues can potentially be solved in a meta-analysis, that is not attempted in this review given the high inhomogeneities among the different studies included.

Additionally, all the selected studies included in the review were carried out in countries were a CXR at entry is currently performed for active TB screening of newly arrived migrants. Most of the papers aimed at defining cost-effectiveness of adding screening for LTBI to the 
ongoing CXR-based screening for active TB $[15,18-21,23]$. The cost-effectiveness of LTBI screening of migrants population should also be evaluated in other low endemic countries.

While most of the studies found that screening programs for detecting and treating LTBI lead to substantial health and economic benefits under a societal perspective, other studies reached opposite conclusions. The importance of how methodological differences affect results of EEs has been recently demonstrated in a systematic review on methodological aspects of costeffective analysis of IGRAs for the diagnosis of LTBI [24]. According to this study, some of the most relevant contributing factors generating different conclusions are the study inputs selection, the inconsistencies in the costing approach, the utility of the QALY (Quality Adjusted Life Year) as the effectiveness outcome, and the manner in which authors choose to present and interpret study results.

Among the studies selected in our review, Oxlade $\mathrm{O}$ et al. state that screening for active TB with CXR would be the most cost-effective and QFT the least costeffective for screening of migrants on arrival [11], while Pareek $\mathrm{M}$ et al. concluded that mandatory CXR on arrival could be safely eliminated in order to improve screening cost-effectiveness with single-step IGRA [16]. Opposite conclusions were reached because Oxlade $\mathrm{O}$ et al. assumed a very low prevalence of LTBI in arriving immigrants (0.08-2.1\%) [11], while Pareek $\mathrm{M}$ et al. assumed a high rate of LTBI treatment completion ( $85 \%$ in case base scenario) [16].

Studies performed under program conditions have shown that completion of LTBI treatment could be much lower that what was assumed in the study published by Pareek $M$ et al. [16] with a better trend observed when shorter and unsupervised regimen are used [25]. It will be interesting to evaluate the completion rate and cost-effectiveness of shorter regimen such as weekly-administered rifapentine plus isoniazid for three month $[26,27]$ in migrants.

In migrants the diagnostic delay in cases of active $\mathrm{TB}$ is mainly related to the delayed presentation of the patients to the health system $[28,29]$ and it is responsible for the spread of the disease among other members of their community. Contact investigation should therefore be strengthened in migrants, as well as in the general population [30]. However, as assumed by the majority of economic models reviewed, screening and treatment for LTBI in migrants would prevent active TB cases and solve the problem at its roots.

The reliability of Markov models, where time horizons of 20 years or life time were used, may be matter of concern. In fact, in most cases, modeling was based on published or retrospective data while the prospective trials supporting three EEs were all open label and only one was controlled. The actual epidemiological data shows that the global TB burden is reducing, though at a slow pace, at global level [31], and in industrialized countries, particularly among native populations [32]. In this scenario, while the yield of the screening for LTBI will decrease over time, however the contribution to the reduction of incidence by diagnosis and treatment of LTBI will progressively increase.

Growing consensus indicates that progress in TB control in the low- and middle-income world will require not only investment in strengthening TB control programs, diagnostics, and treatment but also action on the social determinants of the disease [33]. To reduce the incidence of TB, the drivers of the epidemic and social determinants of TB need to be addressed. These include co-morbidities, substance use, the social and economic conditions that determine both the course of the TB epidemic and exposure to these risk factors [34]. This is probably true for control of TB in migrants that often live in disadvantaged socio-economic conditions in the host country with an increased risk of both, to reactivate LTBI or to acquire a new infection. In this perspective LTBI screening and treatment in migrants could represent a synergistic tool to achieve the ambitious aim of TB elimination.

\section{Conclusions}

The majority of the studies support the use of LTBI screening strategies in migrants based on their costeffectiveness findings. When LTBI screening for migrants are implemented they should focus especially on young migrants from high incidence countries and effort should be done to maximize the adherence to LTBI treatment. In this view shorter LTBI treatment regimen are preferred. Based on our review of EE analysis studies, the use of one step IGRA is the best option in this particular setting. These findings should be confirmed by a cost-effectiveness evaluation based on a medium-term prospective study.

LTBI should be well integrated among the TB control program for migrants and must be part of a wider approach with the aim of facilitating access of migrants to the national health system, re-orienting health services, improving the adhesion to anti-tuberculosis treatment in cases of active TB, and promoting early diagnosis of active TB cases by primary care health operators [35].

\section{Additional file}

Additional file 1: Table S1. Different assumptions concerning progression rate and sensitivity as well as specificity of the TST and IGRAs applied in the different papers. Table S2. Clinical trials referring to economic evaluations. (DOCX 22 kb) 


\section{Competing interests}

The authors declare that they have no competing interests.

\section{Authors' contributions}

Conceived and designed the study: AB, FB, EG, MB. Performed the study: LZ, GC, MS, CL. Analyzed the data: LZ, GC, MB. Wrote the paper: LZ, GC, MB, AM, $A B$. All authors read and approved the final manuscript.

\section{Authors' information}

Not applicable.

\section{Acknowledgments}

Authors thank the COHEMI (COordinating resources to assess and improve HEalth status of Mlgrants from Latin America)-project study group that includes: Maurizio Bonati ${ }^{a}$, Chiara Pandolfini ${ }^{a}$, Francesca Severino ${ }^{a}$, Valeria Confalonieri ${ }^{a}$, Gianni Tognoni ${ }^{a}$, Zeno Bisoffi ${ }^{b}$, Dora Buonfrate ${ }^{b}$, Andrea Angheben $^{b}$, Marco Albonico ${ }^{b, c}$, Alessandro Bartoloni ${ }^{d}$, Marianne Strohmeyer ${ }^{d}$, Lorenzo Zammarchi ${ }^{d}$, Filippo Bartalesie , Jose Muñoz ${ }^{f}$, Ana Requena-Mendez ${ }^{f}$, Maria Roura ${ }^{f}$, LaiaVentura ${ }^{f}$, Robert Pool ${ }^{g}$, Christopher Pell ${ }^{9}$, Anita Hardon ${ }^{g}$, Peter Chiodinij, Juan Moreirak, Mariella Anselmi ${ }^{k}$, Roberto Sempértegui ${ }^{k}$, Eduardo Gotuzzo', Maria Alejandra Mena', Carola Liendo', Héctor H. Garciam, Javier Bustos ${ }^{m}$, Saul Santivañez ${ }^{m}$, Faustino Torricon ${ }^{n}$, Daniel Lozanon, Teresa Hinojosa Cabrera ${ }^{\circ}$, Javier Ochoa Morón ${ }^{\circ}$, Ignacio AbaporiCuellar ${ }^{\circ}$, Jaime Amorós Suarez ${ }^{\circ}$, Guido ChumirayRojas ${ }^{\circ}$, Alessandra Nicoletti ${ }^{p}$, Elisa Bruno ${ }^{p}$. a) Department of Public Health, Laboratory for Mother and Child Health, IRCCS-Istituto di RicercheFarmacologiche Mario Negri, Milan, Italy.

b) Centre for Tropical Diseases, SacroCuore-Don Calabria Hospital, Negrar, Verona, Italy.

c) Ivo de Carneri Foundation, Milano, Italy.

d) Infectious Disease Unit, Department of Experimental \& Clinical Medicine,

University of Florence School of Medicine, Florence, Italy.

e) SOD Malattie Infettive e Tropicali, Azienda Ospedaliero-Universitaria Careggi, Florence, Italy.

f) Servicio de Medicina Tropical y Saludlnternacional, Centre de Recerca en SalutInternacional de Barcelona, Hospital Clínic-Universitat de Barcelona, Barcelona,Spain

g) Centre for Social Science and Global Health, University of Amsterdam, The Netherlands.

j) Hospital for Tropical Diseases and London School of Hygiene \& Tropical Medicine, London, United Kingdom.

k) Centre for Community Epidemiology and Tropical Medicine, Esmeraldas, Ecuador.

I) Instituto de Medicina Tropical Alexander von Humboldt, Universidad Cayetano Heredia, Lima, Peru.

m) Cysticercosis Unit, Instituto de CienciasNeurologicas, Department of Microbiology, Universidad PeruanaCayetano Heredia, Lima, Peru.

n) Colectivo de EstudiosAplicados y Desarrollo Social, Cochabamba, Bolivia.

o) Taller de Educacion y Comunicacion TEKO-GUARANI, Camiri, Bolivia.

p) Dipartimento G.F. Ingrassia Sezione di Neuroscienze, Università di Catania, Catania, Italy.

\section{Availability of data and materials}

Not applicable.

\section{Funding source}

This work has been supported by the European Commission within the 7th Framework Programme under the COHEMI (COordinating resources to assess and improve HEalth status of Mlgrants from Latin America)-project - grant agreement no. FP7-GA-261495.

\section{Author details}

${ }^{1}$ Infectious Diseases Unit, Department of Experimental \& Clinical Medicine, University of Florence School of Medicine, Largo Brambilla 3, 50134 Florence, Italy. ${ }^{2}$ Laboratory for Mother and Child Health, Department of Public Health, IRCCS - Istituto di Ricerche Farmacologiche Mario Negri, Via G. La Masa 19, 20156 Milan, Italy. ${ }^{3}$ SOD Malattie Infettive e Tropicali, Azienda Ospedaliero-Universitaria Careggi, Largo Brambilla 3, 50134 Florence, Italy. ${ }^{4}$ Instituto de Medicina Tropical "Alexander von Humboldt", Universidad Peruana Cayetano Heredia, Barrios Altos, Lima, Peru. ${ }^{5}$ Institute of Infectious and Tropical Diaseases, WHO Collaborting Centre for TB Co-infection and TB Elimination, University of Brescia, Brescia, Italy.
Received: 16 November 2014 Accepted: 7 September 2015

Published online: 24 September 2015

\section{References}

1. European Centre for Disease Prevention and Control/WHO Regional Office for Europe. Tuberculosis surveillance and monitoring in Europe 2012. 2012.

2. Klinkenberg E, Manissero D, Semenza JC, Verver S. Migrant tuberculosis screening in the EU/EEA: yield, coverage and limitations. Eur Respir J. 2009;34(5):1180-9.

3. Codecasa LR, Porretta AD, Gori A, Franzetti F, Degli Esposti A, Lizioli A, et al. Tuberculosis among immigrants from developing countries in the province of Milan, 1993-1996. Int J Tuberc Lung Dis. 1999:3(7):589-95.

4. Lillebaek T, Andersen AB, Bauer J, Dirksen A, Glismann S, de Haas P, et al. Risk of Mycobacterium tuberculosis transmission in a low-incidence country due to immigration from high-incidence areas. J Clin Microbiol. 2001;39(3):855-61.

5. Diel R, Rusch-Gerdes S, Niemann S. Molecular epidemiology of tuberculosis among immigrants in Hamburg, Germany. J Clin Microbiol. 2004;42(7):2952-60.

6. Lobato MN, Hopewell PC. Mycobacterium tuberculosis infection after travel to or contact with visitors from countries with a high prevalence of tuberculosis. Am J Respir Crit Care Med. 1998;158(6):1871-5.

7. Pareek M, Baussano I, Abubakar I, Dye C, Lalvani A. Evaluation of immigrant tuberculosis screening in industrialized countries. Emerg Infect Dis. 2012;18(9):1422-9.

8. Franzetti F, Codecasa L, Matteelli A, Degli Esposti A, Bandera A, Lacchini C, et al. Genotyping analyses of tuberculosis transmission among immigrant residents in Italy. Clin Microbiol Infect. 2010;16(8):1149-54.

9. Hernandez-Garduno E, Kunimoto D, Wang L, Rodrigues M, Elwood RK, Black W, et al. Predictors of clustering of tuberculosis in Greater Vancouver: a molecular epidemiologic study. CMAJ. 2002;167(4):349-52.

10. Alvarez GG, Gushulak B, Abu Rumman K, Altpeter E, Chemtob D, Douglas P, et al. A comparative examination of tuberculosis immigration medical screening programs from selected countries with high immigration and low tuberculosis incidence rates. BMC Infect Dis. 2011;11:3.

11. Oxlade $O$, Schwartzman $K$, Menzies $D$. Interferon-gamma release assays and TB screening in high-income countries: a cost-effectiveness analysis. Int J Tuberc Lung Dis. 2007;11(1):16-26.

12. Broekmans J, Migliori G, Rieder H, Lees J, Ruutu P, Loddenkemper R, et al. European framework for tuberculosis control and elimination in countries with a low incidence. Recommendations of the World Health Organization (WHO), International Union Against Tuberculosis and Lung Disease (IUATLD) and Royal Netherlands Tuberculosis Association (KNCV) Working Group. Eur Respir J. 2002;19(4):765-75.

13. World Health Organization. Global strategy and targets for tuberculosis prevention, care and control after 2015. Geneva: World Health Organization; 2014. EB134R4.

14. Haycox A, Walley T. Pharmacoeconomics: evaluating the evaluators. Br J Clin Pharmacol. 1997:43:451-6.

15. Pareek M, Watson JP, Ormerod LP, Kon OM, Woltmann G, White PJ, et al. Screening of immigrants in the UK for imported latent tuberculosis: a multicentre cohort study and cost-effectiveness analysis. Lancet Infect Dis. 2011;11(6):435-44.

16. Pareek M, Bond M, Shorey J, Seneviratne S, Guy M, White P, Lalvani A, Kon OM. Community-based evaluation of immigrant tuberculosis screening using interferon $\gamma$ release assays and tuberculin skin testing:observational study and economic analysis. Thorax. 2013 Mar;68(3):230-9. doi:10.1136/ thoraxjnl-2011-201542. Epub 2012.

17. Brassard P, Steensma C, Cadieux L, Lands LC. Evaluation of a school-based tuberculosis-screening program and associate investigation targeting recently immigrated children in a low-burden country. Pediatrics. 2006;117(2):e148-56.

18. Khan K, Muennig P, Behta M, Zivin JG. Global drug-resistance patterns and the management of latent tuberculosis infection in immigrants to the United States. N Engl J Med. 2002;347(23):1850-9.

19. Dasgupta K, Schwartzman K, Marchand R, Tennenbaum TN, Brassard P, Menzies D. Comparison of cost-effectiveness of tuberculosis screening of close contacts and foreign-born populations. Am J Respir Crit Care Med. 2000;162(6):2079-86.

20. Porco TC, Lewis B, Marseille E, Grinsdale J, Flood JM, Royce SE. Costeffectiveness of tuberculosis evaluation and treatment of newly-arrived immigrants. BMC Public Health. 2006;6:157. 
21. Hardy AB, Varma R, Collyns T, Moffitt SJ, Mullarkey C, Watson JP. Cost-effectiveness of the NICE guidelines for screening for latent tuberculosis infection: the QuantiFERON-TB Gold IGRA alone is more cost-effective for immigrants from high burden countries. Thorax. 2010;65(2):178-80.

22. Iqbal AZ, Leighton J, Anthony J, Knaup RC, Peters EB, Bailey TC. Costeffectiveness of using Quantiferon Gold (QFT-G)(R) versus tuberculin skin test (TST) among U.S. and foreign born populations at a public health department clinic with a low prevalence of tuberculosis. Public Health Nurs (Boston, Mass). 2014;31(2):144-52.

23. Linas BP, Wong AY, Freedberg KA, Horsburgh Jr CR. Priorities for screening and treatment of latent tuberculosis infection in the United States. Am J Respir Crit Care Med. 2011;184(5):590-601.

24. Oxlade O, Pinto M, Trajman A, Menzies D. How methodologic differences affect results of economic analyses: a systematic review of interferon gamma release assays for the diagnosis of LTBI. PLoS One. 2013;8(3), e56044.

25. Matteelli A, Casalini C, Raviglione MC, El-Hamad I, Scolari C, Bombana E, et al. Supervised preventive therapy for latent tuberculosis infection in illegal immigrants in Italy. Am J Respir Crit Care Med. 2000;162(5):1653-5.

26. Sterling T, Munsiff SS, Frieden TR. Management of latent tuberculosis infection in immigrants. N Engl J Med. 2003;348(13):1289-92. author reply 1289-1292.

27. Shepardson D, Marks SM, Chesson H, Kerrigan A, Holland DP, Scott N, et al. Cost-effectiveness of a 12-dose regimen for treating latent tuberculous infection in the United States. Int J Tuberc Lung Dis. 2013;17(12):1531-7.

28. Gagliotti C, Resi D, Moro ML. Delay in the treatment of pulmonary TB in a changing demographic scenario. Int J Tuberc Lung Dis. 2006;10(3):305-9.

29. Mor Z, Kolb H, Lidji M, Migliori G, Leventhal A. Tuberculosis diagnostic delay and therapy outcomes of nonnational migrants in Tel Aviv, 1998-2008. Euro Surveill. 2013;18(12). pii: 20433.

30. Fox GJ, Barry SE, Britton WJ, Marks GB. Contact investigation for tuberculosis: a systematic review and meta-analysis. Eur Respir J. 2013;41(1):140-56.

31. World Health Organization. Global tuberculosis control 2011. 2011.

32. European Centre for Disease Control and Prevention, World Health Organization Regional Office for Europe. Tuberculosis surveillance and monitoring in Europe 2012. In: Surveillance report. Stockholm: European Centre for Disease Control and Prevention; 2012. p. 73-97.

33. Hargreaves JR, Boccia D, Evans CA, Adato M, Petticrew M, Porter JD. The social determinants of tuberculosis: from evidence to action. Am J Public Health. 2011;101(4):654-62.

34. Rasanathan K, Sivasankara Kurup A, Jaramillo E, Lonnroth K. The social determinants of health: key to global tuberculosis control. Int J Tuberc Lung Dis. 2011;15 Suppl 2:S30-6.

35. Ministero della Salute. Aggiornamento delle raccomandazioni per le attività di controllo della tubercolosi. "Politiche efficaci a contrastare la tubercolosi nella popolazione immigrata". 2010.

\section{Submit your next manuscript to BioMed Central and take full advantage of:}

- Convenient online submission

- Thorough peer review

- No space constraints or color figure charges

- Immediate publication on acceptance

- Inclusion in PubMed, CAS, Scopus and Google Scholar

- Research which is freely available for redistribution 